\title{
EVALUATION OF THE REMINERALIZING EFFECT OF FLUORIDATED TOOTHPASTE WITH SODIUM HEXAMETAPHOSPHATE ON DEMINERALIZED PRIMARY TEETH ENAMEL
}

Mohamed AboAlFtooh Shehata", Nagwa Mohammed Khattab ${ }^{* *}$ and Mina Kamal Yassa ${ }^{* * * *}$

\begin{abstract}
Background: A primary aim of pediatric dentists is to repair the early demineralized enamel without intervention through remineralization. Sodium hexametaphosphate (SHMP) is a remineralizing material that has been reported to have promising results for caries management.
\end{abstract}

Aim of the study: Evaluation of the remineralization potential of fluoridated toothpaste with SHMP on demineralized enamel of primary teeth.

Materials and Methods: Fifty six freshly extracted anterior deciduous teeth were collected and divided into four groups regarding the concentrations of SHMP added to $400 \mathrm{ppm}$ F containing tooth paste. Three groups contained $(0.25 \%, 0.5 \%, 1 \%)$ respectively and a control group without SHMP. Specimens were treated with the tooth pastes and exposed to $\mathrm{pH}$ cycling for 5 days then a remineralizing solution for additional 2 days. Specimens were evaluated using Digital Radiographic Density Analysis at baseline, after demineralization and after remineralization.

Results: Teeth treated with SHMP showed marked remineralization effect than those treated with fluoride alone. Increasing the concentration of SHMP from $0.25,0.5$ to $1 \%$, associated with increased the remineralizing effect.

Conclusion: SHMP has a superior remineralizing effect over fluoride alone. 1\% SHMP concentration added to $400 \mathrm{~F}$ ppm toothpaste provided the best results.

KEY WORDS: Enamel remineralization, Sodium Hexametaphosphate, Toothpaste, Fluoride, Primary teeth.

\footnotetext{
* Demonstrator in Pediatric and Community Dentistry Department, Faculty of Dentistry, Minia University.

** Professor in Pediatric Dentistry and Dental Public Health, Faculty of Dentistry, Ain Shams University.

*** Lecturer in Pediatric and Community Dentistry Department, Faculty of Dentistry, Minia University.
} 


\section{INTRODUCTION}

Despite the efforts of preventive dentistry, dental caries still has high prevalence. Remineralization aims to repair the early demineralized enamel lesions without intervention to prevent caries progression and cavitation ${ }^{(1)}$. Remineralization redeposits minerals lost by enamel. It occurs by deposition of calcium and phosphate on tooth surface therefore, preventing early enamel lesions ${ }^{(2)}$.

Fluoride can promote remineralization and inhibit demineralization of enamel ${ }^{(3,4)}$. However, increasing concerns regarding safety of products with high fluoride concentration have evolved ${ }^{(5)}$.

SHMP is a remineralizing material that has reported promising results for caries management. In addition, it seems that remineralizing agents exert synergic effects when used together with SHMP ${ }^{(6)}$.

The current study was conducted to evaluate the effect of remineralization potential of fluoridated hexametaphosphate toothpaste on primary demineralized tooth enamel using Direct Digital Radiographic Density Analysis.

\section{MATERIALS AND METHODS}

\section{Teeth collection}

Fifty six freshly extracted anterior deciduous teeth were collected, and stored in physiologic saline for maximum one month. Selected teeth were sound; free of caries, restoration, cracks, white spots or enamel defects.

\section{Teeth preparation}

Teeth were manually scaled, cleaned by soft brush with non-fluoridated toothpaste, washed and stored in physiologic saline in closed container. A piece of adhesive tape $(3 \times 3 \mathrm{~mm})$ was put on the center of labial surfaces, and then nail polish was applied on the surface to standardize the area of treatment and evaluation.

\section{Grouping}

Thereafter, teeth were randomly allocated in equal 4 groups. Group (1) with no SHMP, group (2) with $0.25 \%$ SHMP, group (3) with $0.5 \%$ SHMP and group (4) with $1 \%$ SHMP.

The concentration of SHMP was adjusted by adding $0.25 \mathrm{gm}, 0.5 \mathrm{gm}$ and $1 \mathrm{gm}$ to a $100 \mathrm{ml}$ of $400 \mathrm{ppm}$ of the fluoridated toothpaste to be ready for brushing.

\section{Demineralization}

Based on da Camara et al.(2016) ${ }^{(7)}$, Sub-surface artificial caries was created on enamel specimens by placing them solely for 72 hour at $37^{\circ} \mathrm{C}$ in $7.0 \mathrm{ml}$. of a demineralizing solution $(2.2 \mathrm{mM} \mathrm{CaCl}, 2.2 \mathrm{mM}$ $\mathrm{NaH}_{2} \mathrm{PO}_{4}$, and $50 \mathrm{Mm}$ acetic acid, adjusted $\mathrm{pH}$ of 4.7 with $1 \mathrm{M} \mathrm{KOH})$.

Samples were exposed to $\mathrm{pH}$ cycles for 5 days, each cycle involved six hours of demineralization (two hours each, three times daily), with eight hours of remineralization in between(four hours each, twice daily). A one-minute treatment (by brushing the teeth with the toothpaste) was given before the remineralization cycle three times daily. Then, all the samples were placed in remineralizing solution overnight at $37^{\circ} \mathrm{C}$ in an incubator for 10 hours. Finally, Samples were dipped for 2 additional days in a remineralization solution $(2.00 \mathrm{~g} / \mathrm{L}$ methyl p-hydroxybenzoate, $10.0 \mathrm{~g} / \mathrm{L}$ sodium carboxymethyl cellulose, $8.38 \mathrm{mmol} / \mathrm{L} \mathrm{KCl}, 0.29 \mathrm{mmol} / \mathrm{L}$ $\mathrm{MgCl} 2.6 \mathrm{H} 2 \mathrm{O}, 1.13 \mathrm{mmol} / \mathrm{L} \mathrm{CaCl} 2.2 \mathrm{H} 2 \mathrm{O}, 4.62$ $\mathrm{mmol} / \mathrm{L} \mathrm{KH} 2 \mathrm{PO} 4,2.40 \mathrm{mmol} / \mathrm{L} \mathrm{K} 2 \mathrm{HPO} 4$; and adjusted $\mathrm{pH}$ was 7.0 using $\mathrm{KOH})$ to simulate saliva ${ }^{(8,9)}$.

Samples analysis was done using Direct Digital Radiographic Density Analysis by a computer program calculated automatically mean gray level values using direct digital radiographic imaging system (Digora unit Orion Crpp. Sordex medical systems, Helsinki, Finlan). All the prepared specimens were digitally radiographed before demineralization, after demineralization and after treatment. Treatment effect was manifested radiographically as a change in pixel gray scale values (radio density) from their initial records. 
The data were collected and the mean and standard deviation of grey shade values were calculated for each group in each test. Kolmogorov-Smirnov and Shapiro-Wilk tests explored the normality of data showing parametric distribution.

One-way ANOVA followed by Tukey post-hoc test was used to compare between more than two groups in non-related samples while repeated measure ANOVA was used to compare between more than two groups in related samples. Two groups in related samples were compared using paired sample t-test and the significance level was set at $\mathrm{P} \leq$ 0.05 . Statistical analysis was performed with IBM $®$ SPSS ${ }^{\circledR}$ Statistics Version 20 for Windows.

\section{RESULTS}

In each tested group, there was a statistically significant difference before demineralization, after demineralization and after remineralization where $(\mathrm{p}<0.001)$.

Regarding relation between groups, No statistically significant difference $(\mathrm{p}=1)$ was detected before and after demineralization.

However, after remineralization, it was found that the mean grey values increased,due to the increase in the mineral content, with the increase of SHMP concentration and there was a statistically significant difference between the tested groups where $(\mathrm{p}<0.001)$. Figures $(\mathbf{1 , 2})$

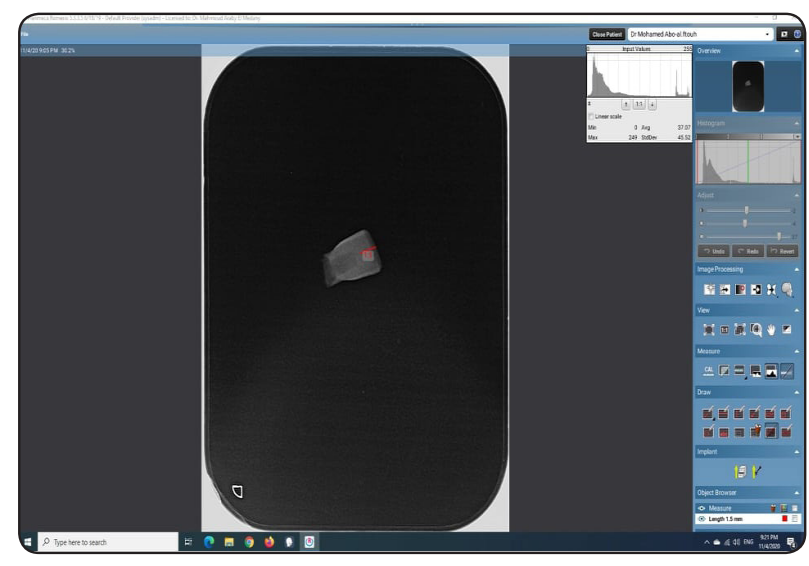

Fig. (1): The mean of grey shade value of the marked area.

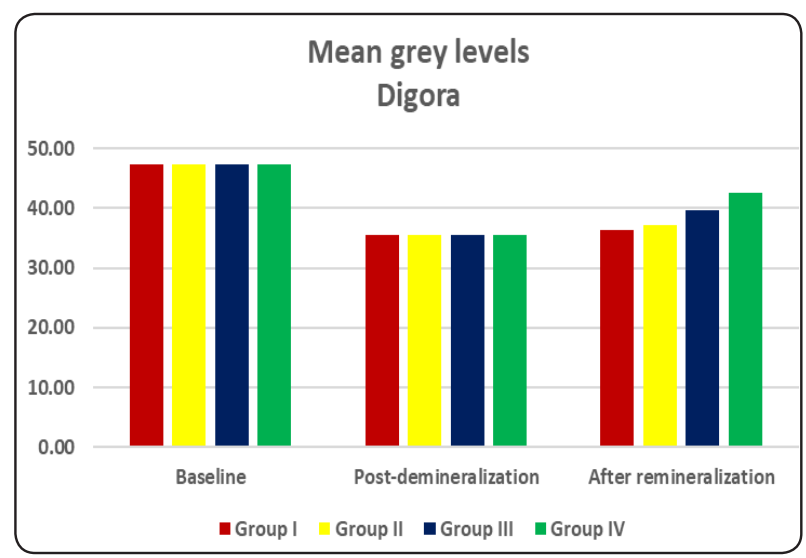

Fig. (2): Bar chart representing mean grey levels for the different groups.

\section{DISCUSSION}

Remineralization constitutes an effective method for minimally invasive dentistry that redeposits minerals lost from enamel preventing caries progression. While fluoride gains much popularity in remineralization, a number of new remineralization agents have been investigated and commercialized to promote deeper remineralization of early demineralized enamel to facilitate caries control ${ }^{(10)}$.

Fluoridated dentifrices are suggested to be the cornerstone for caries suppression in children. Normally, fluoridated dentifrices contain 1000 ppm fluoride $^{(11)}$, however, due to safety considerations; lower fluoride content tooth pastes have been suggested for children ${ }^{(12)}$.

SHMP is one of the remineralizing materials that were introduced in 2000 as an effective anti-tartar component in toothpastes ${ }^{(13)}$.

Since the applications and concentrations of remineralizing agents differ from child to adult and that remineralizing agents exert synergic effects when used together ${ }^{(1)}$, this study was performed to evaluate the remineralization effect of fluoridated toothpaste with SHMP on enamel of primary teeth and to find the best concentration.

This study was designed as in vitro study on deciduous teeth using $\mathrm{pH}$ cycling model that involved alternating the application of demineralization 
and remineralization solutions at constant time intervals in order to simulate $\mathrm{pH}$ cycles in the oral environment ${ }^{(14)}$.

Specimens were evaluated using Digital Radiographic Density Analysis. The changes from demineralization and remineralization can be detected from density value ${ }^{(15)}$. Measuring the average gray level values was sensitive enough to detect small changes in the enamel surface ${ }^{(16) .}$

The data gathered from this study was consistent with da Camara et al. (2016), who added SHMP to 1100-ppm F t oothpaste and found marked remineralizati o $\mathrm{n}$ of the demineralized enamel. Moreover, when the concentration increased from $0.25,0.5$ and $1 \%$ of SHMP the remineralization power increased ${ }^{(7)}$.

However, highe $\mathrm{r}$ concentrations above $1 \%$ SHMP were not tested in the current study since these concentrations were detrimental, as they could interfere with absorption of fluoride into the enamel, larger percent of SHMP can sequester calcium ions from hydroxyapatite ${ }^{(7)}$

\section{CONCLUSION}

1) Adding SHMP to a $400 \mathrm{ppm}$ flouride tooth paste promoted the remineralizing potential over fluoride alone.

2) The best concentration for $400 \mathrm{~F} \mathrm{ppm}$ toothpaste is $1 \%$ SHMP.

\section{REFERENCES}

1. Cochrane NJ, Cai F, Huq NL, Burrow MF, Reynolds EC. New approaches to enhanced remineralization of tooth enamel. J Dent Res. 2010 Nov; 89(11):1187-1197.

2. Abou Neel EA, Aljabo A, Strange A, Ibrahim S, Coathup M, Young AM, Bozec L, Mudera V. Demineralizationremineralization dynamics in teeth and bone. Int J Nanomedicine. 2016 Sep 19;11:4743-4763.

3. Thurnheer T, Belibasakis GN. Effect of sodium fluoride on oral biofilm microbiota and enamel demineralization. Arch Oral Biol. 2018 May;89:77-83.

4. Buzalaf MAR, Pessan JP, Honório HM, Ten Cate JM. Mechanisms of action of fluoride for caries control. Monogr Oral Sci. 2011;22:97-114.
5. Zohoori FV, Maguire A. Are there good reasons for fluoride labelling of food and drink? Br Dent J. 2018 Feb 23; 224(4):215-217.

6. Mohammadipour HS, Maghrebi ZF, Ramezanian N, Ahrari F, Daluyi RA. The effects of sodium hexametaphosphate combined with other remineralizing agents on the staining and microhardness of early enamel caries: An in vitro modified pH-cycling model. Dent Res J (Isfahan). 2019 Nov 12; 16(6):398-406.

7. da Camara DM, Pessan JP, Francati TM, Souza JA, Danelon M, Delbem AC. Fluoride toothpaste supplemented with sodium hexametaphosphate reduces enamel demineralization in vitro. Clin Oral Investig. 2016 Nov; 20(8):1981-1985.

8. da Camara DM, Miyasaki ML, Danelon M, Sassaki KT, Delbem AC. Effect of low-fluoride toothpastes combined with hexametaphosphate on in vitro enamel demineralization. J Dent. 2014 Mar; 42(3):256-262.

9. da Camara DM, Pessan JP, Francati TM, Santos Souza JA, Danelon M, Delbem AC. Synergistic effect of fluoride and sodium hexametaphosphate in toothpaste on enamel demineralization in situ. J Dent. 2015 Oct; 43(10):12491254.

10. Philip N. State of the Art Enamel Remineralization Systems: The Next Frontier in Caries Management. Caries Res. 2019;53(3):284-295.

11. Cury JA, Tenuta LM. Evidence-based recommendation on toothpaste use. Braz Oral Res. 2014; 28 Spec No: 1-7.

12. Holt RD, Nunn JH, Rock WP, Page J. Fluoride dietary supplements and fluoride toothpastes for children. Int $\mathbf{J}$ Paediatr Dent.1996 Jun; 6: 139-142.

13. Sensabaugh C, Sagel ME. Stannous fluoride dentifrice with sodium hexametaphosphate: review of laboratory, clinical and practice-based data. J Dent Hyg. 2009 Spring; 83(2):70-78.

14. ten Cate JM, Timmer K, Shariati M, Featherstone JD. Effect of timing of fluoride treatment on enamel de- and remineralization in vitro: a pH-cycling study. Caries Res. 1988; 22(1):20-26.

15. Khattab NM, Mahmoud HZ. Effects of laser and fluoride on the acid resistance of human dental enamel of primary molars and premolars. Egyptian Dental Journal, July, 2005; 51:1237-1250.

16. Leite-Ribeiro P, de Oliveira TF, Mathias P, Campo Ede J, Sarmento VA. Conventional and digital radiographic assessment of tooth enamel de-/remineralization processes: an experimental study. Indian J Dent Res. 2014 Mar-Apr; 25(2):214-219. 